\title{
Difficulties in the Construction of Subway Tunnel Underpass Project and Research on Ecological Restoration Technology
}

\author{
Yongchao Zhu ${ }^{1}$, Pengbo Cui ${ }^{2,3}$, Hongshuo Sun ${ }^{1 *}$ \\ ${ }^{1}$ Department of Railway Engineering, Zhengzhou Railway Vocational and Technical College, Zhengzhou, Henan, \\ China; \\ ${ }^{2}$ School of Civil Engineering, Shijiazhuang Tiedao University, Shijiazhuang, China; \\ ${ }^{3}$ Jiangsu Vocational Institute of Architectural Technology, Xuzhou, Jiangsu,China; \\ *Corresponding Author.
}

\begin{abstract}
:
Subway construction is a high-risk construction project, because there are many uncertain factors such as Undercrossing urban hinterland and geological conditions, which lead to the difficulty of organization and management and frequent safety accidents. Therefore, there is an urgent need for effective safety risk analysis model and risk management means to prevent accidents. Aiming at the mechanical problems of surrounding rock and supporting structure of subway tunnel under different construction methods, the dynamic numerical simulation of three pilot tunnel method and upper and lower step method is carried out by using finite element method. The transformation relationship between stress field and displacement field of subway tunnel under different construction procedures is established, and the two construction methods are compared. The test results show that the internal force, axial force and bending moment model of the subway tunnel support structure system established in this paper has a certain guiding significance for the design and safe construction of the subway tunnel.
\end{abstract}

Keywords: Metro Construction, Organization Management, Safety Risk Analysis Model, Ecological Restoration.

\section{INTRODUCTION}

Over the past few decades, the systems that humans have built and the environment in which they have built have been constantly changing. Including the rapid technological change, the continuous change of the nature of the accident, the emergence of new dangers, the increasingly high safety requirements, the increasing complexity and coupling of the system, the more complex man-machine relationship and the continuous updating of the safety concept, these changes have brought many challenges to the safe construction and operation of these systems [1-2]. Therefore, although the level of human science and technology has made great progress in the past few decades, with the continuous innovation of technology, accidents are also happening, such as the Three Mile Island nuclear accident, Chernobyl nuclear accident, Bhopal accident in India, Challenger space shuttle accident and so on [3]. These accidents have caused 
great economic and social impact.

Subway construction has similar experience [4]. On the one hand, it has many uncertainties such as underground city, geological conditions and other uncertain factors, complex technical equipment, long construction cycle and high cost, which makes subway construction very easy to have major safety accidents [5-6]. On the other hand, as a labor intensive industry, organization and management, etc. factors have a key impact on construction safety. For example, the main causes of the accident include over excavation of foundation pit, weak links in steel support system, serious dereliction of duty in monitoring and supervision work [7].

Subway construction is a typical complex social and technical system. The occurrence of subway accidents can be considered as a form of system safety. However, the existing accident models lack a comprehensive analysis of such major accidents from a systematic perspective: on the one hand, the post event analysis of these accidents shows that organization and management factors have an important impact on system safety. On the other hand, the existing accident analysis model for social level analysis such as organization and technical level analysis such as production are often carried out separately, lack of research on the interaction between the two, and the gap between the model and reality makes more and more scholars devote themselves to this. This paper attempts to build a new system security risk analysis model in the field of subway construction from the perspective of complex social technology system, based on system theory and cybernetics.

\section{Feedforward control analysis of shield construction in Metro}

\subsection{Intelligent method of Surface Subsidence Analysis}

Artificial neural network (ANN) is a branch of artificial intelligence research. It is a complex network system formed by a group of artificial neurons with similar structure to human brain neurons. It has good mathematical description, high modeling ability and good data fitting ability for complex nonlinear systems [8].

There are many kinds of artificial neural network models, in which the error back propagation neural network (BPNN) is a common neural network model. BPNN is a multi-layer network structure, including input layer, hidden layer and output layer (see Figure 1). The layers are often connected by full interconnection, and there is no connection between the cells in the same layer [9-109]. The connection strength between neurons is expressed by connection weight. BPNN adjusts the network connection weight by learning the data samples, so as to get the nonlinear mapping relationship between the actual input and output. Because the process of adjusting the connection weight is based on comparing the error between the actual output and the target output of the network, the connection weight is modified from the output layer to the input layer, so it is called back error propagation.

In recent years, artificial neural network has been widely used to solve the deformation analysis caused by deep foundation pit excavation or tunnel excavation. These researches are usually based on BPNN modeling. For example, Kim et al. Used neural network to estimate the maximum settlement value and the reverse bending point of settlement trough caused by tunnel excavation, and the relative connection strength (RSE) in the network was used to identify the most important influencing factors. Neaupane and Adhikari [established two neural network models to predict the maximum ground settlement and horizontal displacement respectively; suwansawat and Einstein analyzed the influence of earth pressure shield 
balanced construction on ground settlement based on neural network modeling, and focused on the relationship between shield parameters and ground settlement.

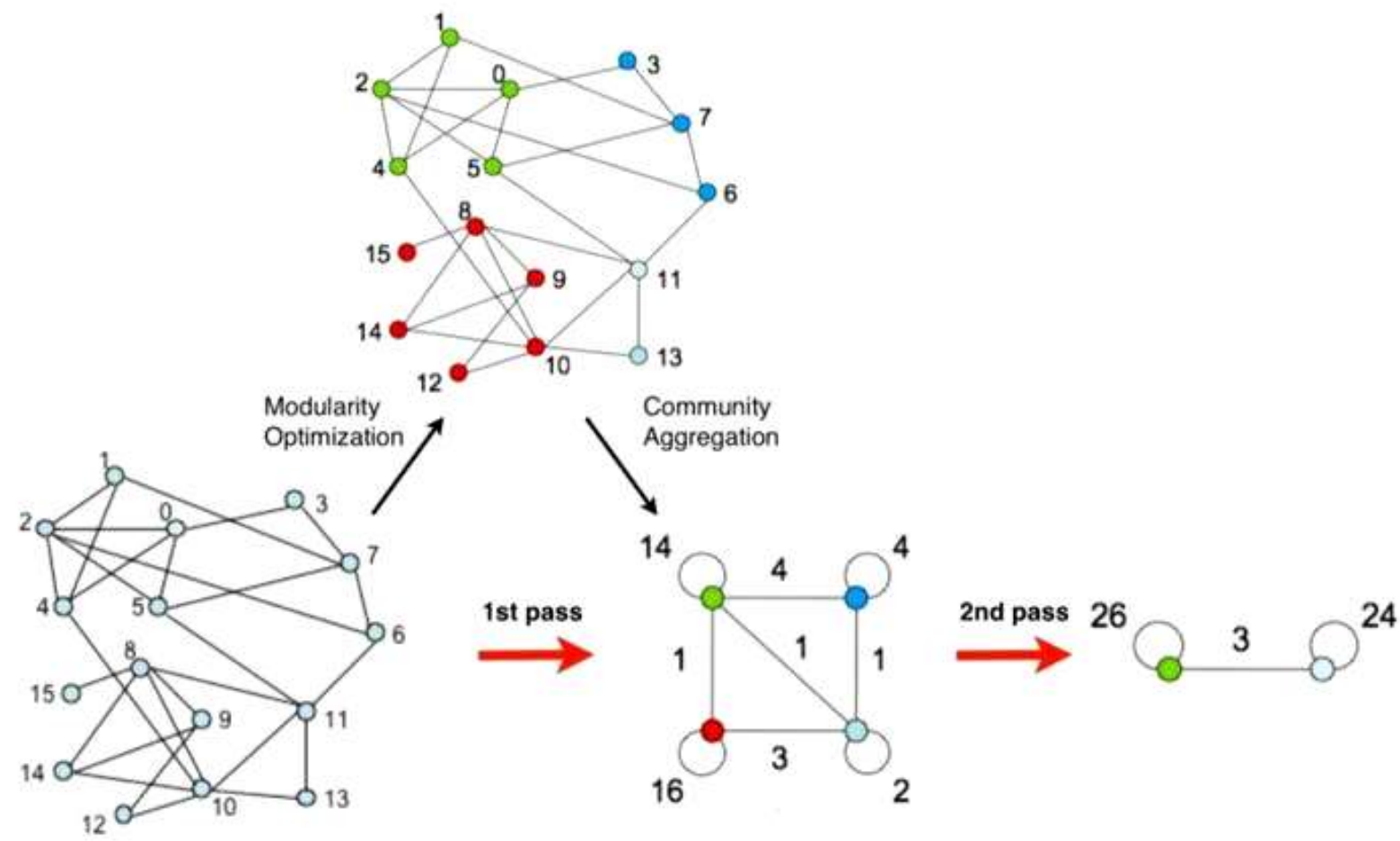

Fig 1: BPNN network structure

\subsection{Intelligent prediction model of surface subsidence}

Most of the existing surface settlement prediction models aim to analyze the maximum settlement of surface settlement, which is more helpful to the decision-making in the design stage than in the construction stage. During the tunnel construction, it is more meaningful to predict the settlement of each excavation step. This is because the construction party can take appropriate measures to control the surface settlement after each excavation step to avoid the occurrence of large settlement. Yeh uses the rolling prediction method to predict the soil pressure of the next excavation by taking the soil pressure of the previous excavation together with other influencing factors as the model input, so as to provide the basis for shield control. Since the monitoring frequency of surface subsidence in this case is once a day, this paper adopts a similar prediction method, that is, one step ahead prediction method, taking the last surface subsidence monitoring value $\mathrm{s}$ and influencing factor $\mathrm{F}$ as the input of the model to predict the next surface subsidence size s' (Fig. 2)

$$
s^{\prime}=f(s, F)
$$

The forecasting model consists of three parts: adaptive Gaussian kernel, sRVM and PSO. the adoption of adaptive Gaussian kernel is an internal mechanism to identify the relative importance of influencing factors. sRVM based on adaptive Gaussian kernel is used to establish the relationship between influencing factors and land subsidence, while PSO is mainly used to find the optimal combination of kernel parameters. 


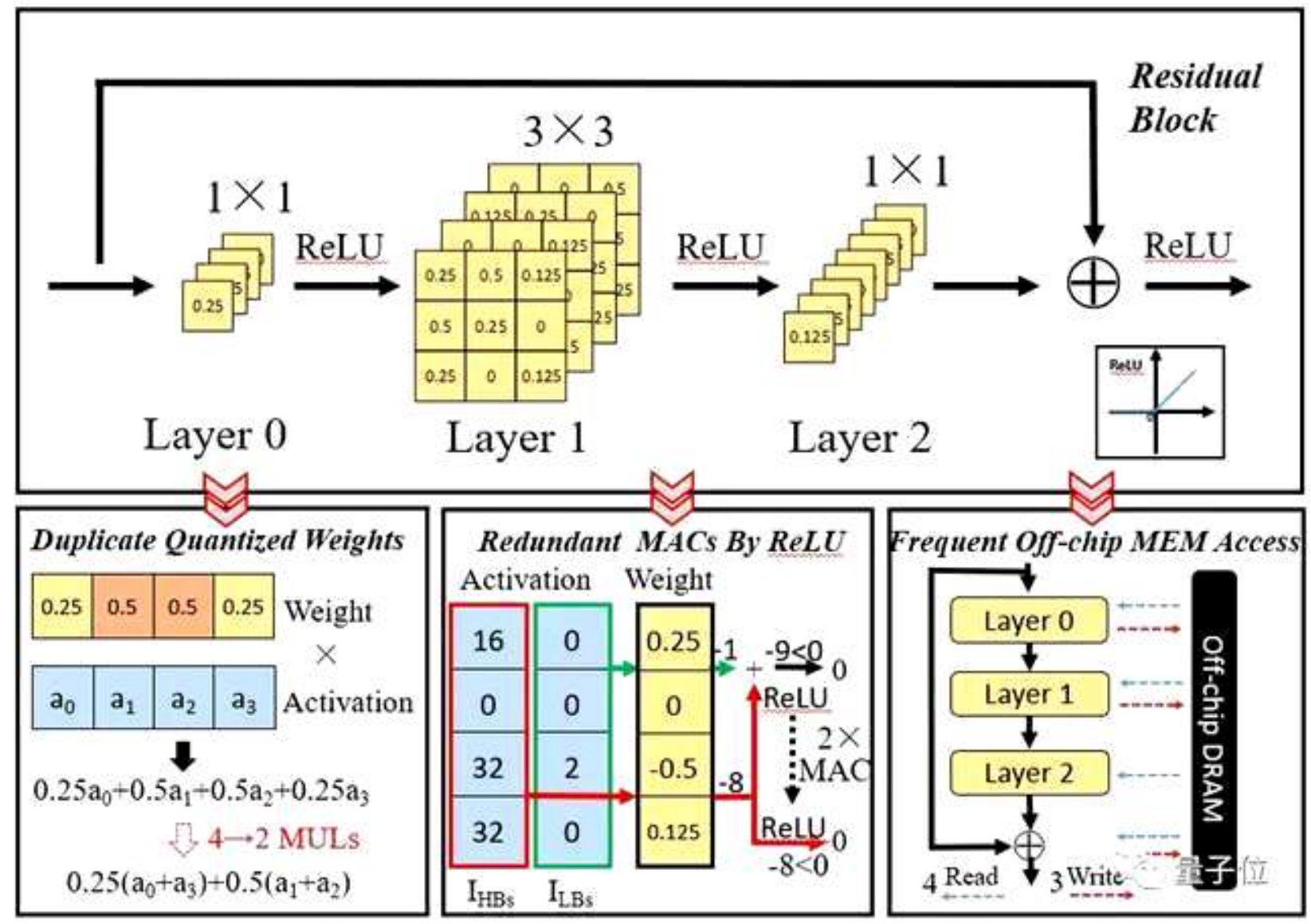

Fig 2: Prediction mode of surface subsidence

\section{HYBRID PROBABILISTIC RISK ASSESSMENT MODEL}

The report of the International Engineering Insurance Association (IAEI) pointed out that most accidents occurred in the tunnel construction stage, and the characteristics of subway construction should be fully considered when evaluating the surrounding environmental risks caused by subway construction. Therefore, the establishment of subway construction risk assessment model should meet the following requirements:

1. The model needs to express the three elements of risk, namely scenario, probability and consequence, which is the basic purpose of modeling;

2. There are many uncertainties in subway construction, including accidental uncertainty and cognitive uncertainty. Using probability to describe can help people understand and deal with this uncertainty. Therefore, the model needs to express the risk propagation in probability.

3. There are many risk factors in subway construction, and zhe IEN pointed out that three conditions need to be met to realize risk control by controlling risk factors. That is to say, all relevant risk influencing factors must be identified, the risk influencing factors must be measurable, and the causal relationship between risk influencing factors and risks is known. Therefore, the model must be able to consider all risk influencing factors of buildings, surrounding roads and surrounding pipelines at the same time, and show the causal relationship between risk influencing factors and risks; 
4. Accidents in subway construction are often not caused by the lack of understanding of unknown factors, but due to the lack of use of existing relevant knowledge. This deficiency is mainly reflected in how to combine historical data and expert judgment, the latter is regarded as a powerful supplement to the former as a kind of knowledge expression, so the model must integrate objective historical data and expert subjective judgment for risk analysis at the same time.

Based on the above analysis, combined with the characteristics of the common risk analysis methods mentioned above, this paper constructs a hybrid probabilistic risk assessment method (see Figure 3), uses FTA / ETA to qualitatively identify possible accident scenarios and risk influencing factors, and constructs the structure of Bayesian network based on this, so as to express the causal relationship between risk influencing factors and risks. Relevance vector classification machine is used to process the historical objective data and transform it into a priori probability table of Bayesian network. Fuzzy theory is used to quantify expert judgment and is used as a conditional probability table of Bayesian network. The final probability and consequence of the accident are deduced by Bayesian network.

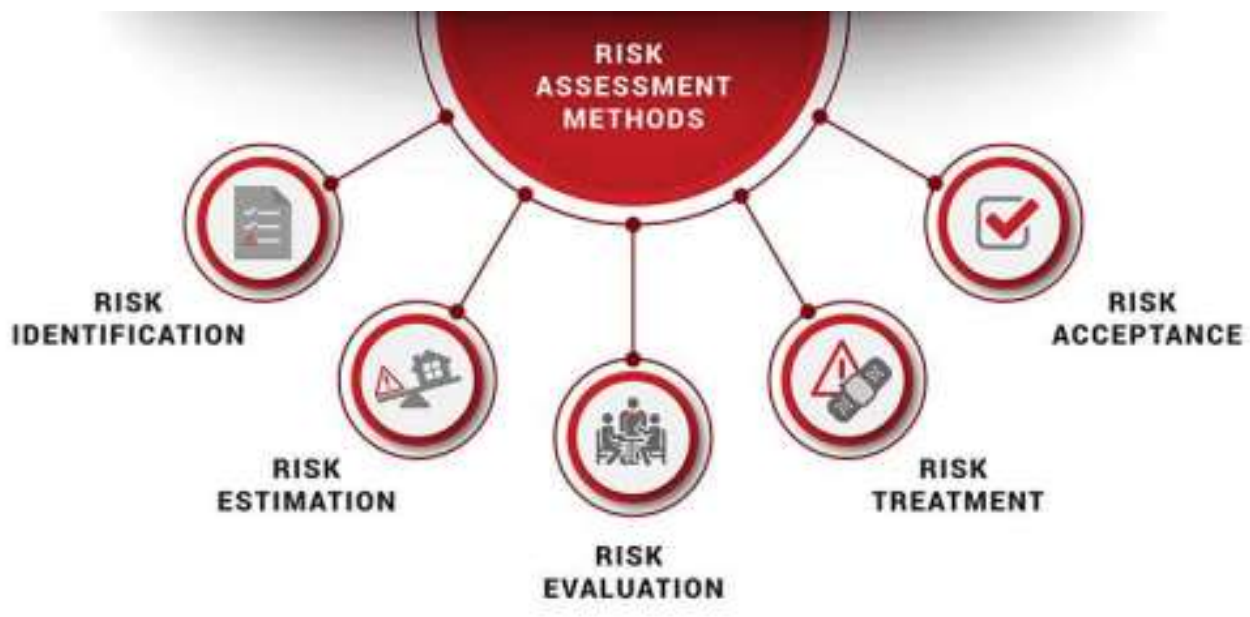

Fig 3: Hybrid of risk assessment methods

\section{ANALYSIS ON THE INFLUENCE OF SUBWAY CONSTRUCTION ORGANIZATION AND MANAGEMENT FACTORS ON SAFETY RISK}

\subsection{Organizational safety performance model}

According to the basic principle a of organization modeling, the purpose of modeling is to analyze the influence of organizational factors on safety risk, while according to Principle B, safety and productivity are the same performance of an organization, and an organization must pursue both production and safety goals. Therefore, the research on the influence of organizational factors on safety risk must be based on the organizational performance model.

At present, safety culture is generally considered to be the root cause of accidents, because it can provide insight into the organization's safety values, that is, the strength of safety awareness. However, the safety culture does not directly determine the safety performance of the organization, but indirectly affects the safety performance of the organization through the safety management system. The safety management system is the performance of the safety culture and reflects the safety management commitment of the 
organization. An efficient safety management system depends on high safety management commitment. Because managers only put safety in a more important position, will give more safety investment, and pay more attention to safety from policy, resources, personnel training and other aspects. Grote and $\mathrm{K}$ ü nzler emphasize that the social technology system model should not only integrate the entity characteristics such as the organization's management mode and process, but also reflect the non entity characteristics such as the organization's beliefs, values and guidelines.

To sum up, this paper will establish an organizational safety performance model based on the framework of "safety culture safety management system safety performance" (see Figure 4).

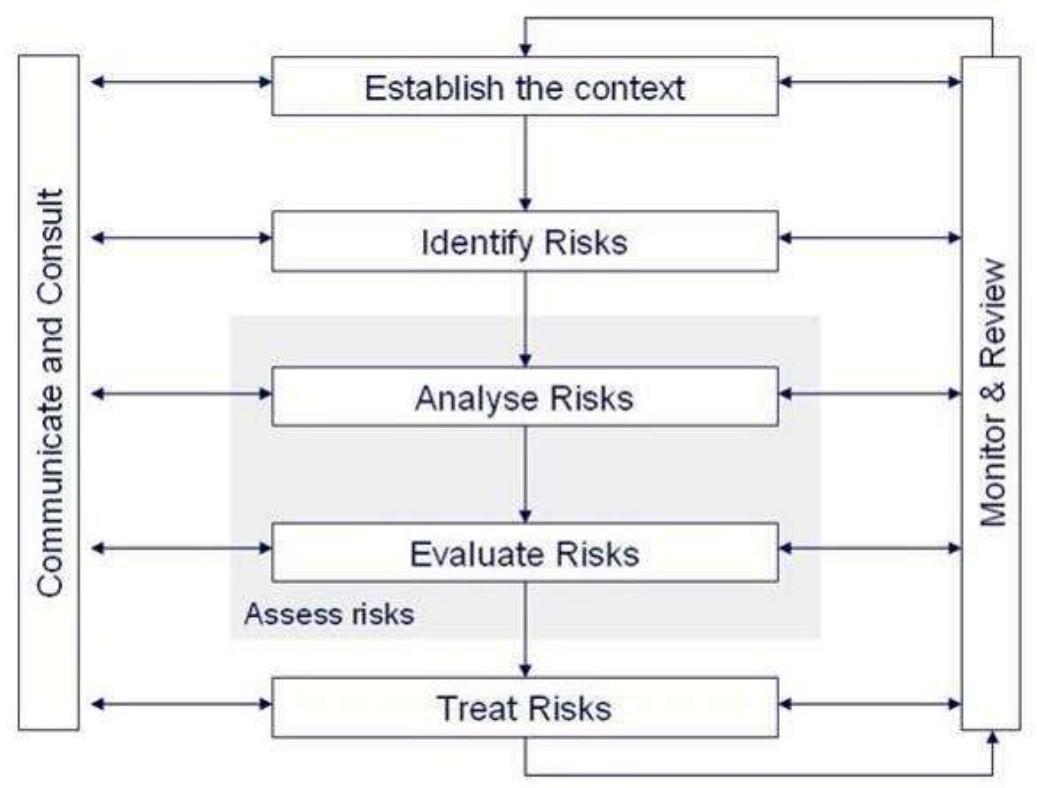

Fig 4: Framework of organizational safety performance model

4.2 The relationship between safety performance and production performance

According to the basic principle B of organization modeling, safety is not the only performance of the organization. For the construction unit, cost and schedule are also very important as the production performance of the organization, and they interact with each other. Organizations need to compensate for the loss caused by safety accidents, which directly affects the cost, and tight funds will also lead to insufficient investment in safety, which makes safety accidents more prone to occur. Safety accidents may also seriously affect the progress of the project, sometimes it needs a certain amount of manpower and material resources to clean up the accident site to resume production, and too fast progress will also distract the attention of the organization on safety and put more into production. However, there is also a relationship of mutual influence and restriction between cost and progress. Lack of funds will slow down the progress of the project. Conversely, to improve the progress, it needs sufficient human, material and financial support.

The research shows that safety culture is the most important organizational factor leading to accidents. Creating a good safety culture can resist or balance the pressure of production. On the one hand, it can reduce the possibility of accidents, on the other hand, it can ensure the continuity of production activities. Subway tunnel construction is a dynamic process. Organizations or managers need to respond to various unplanned events (such as various accidents) in time to balance safety, progress and cost objectives. Safety culture is the key to achieve this delicate balance. Therefore, this paper takes safety culture as the bridge 
between safety and production, and the influence between production and safety will be strengthened or weakened by the role of safety culture.

4.3 Dynamic modeling of organizational performance management system

Kennedy and Kirwan reviewed the assessment methods used from the social level such as organizational management to the technical level such as accident consequences. They summarized these methods into two categories: safety audit method and risk analysis method (as shown in Figure 5). Among them, the safety audit method based on the establishment of various indicators is more suitable for the evaluation of safety culture, safety management and other social factors, but these methods are difficult to get the analysis results of accident consequences. On the contrary, the traditional PRA based risk analysis method can quantitatively analyze the probability and loss of accidents, but it can't bring the remote factors such as safety culture into the model.

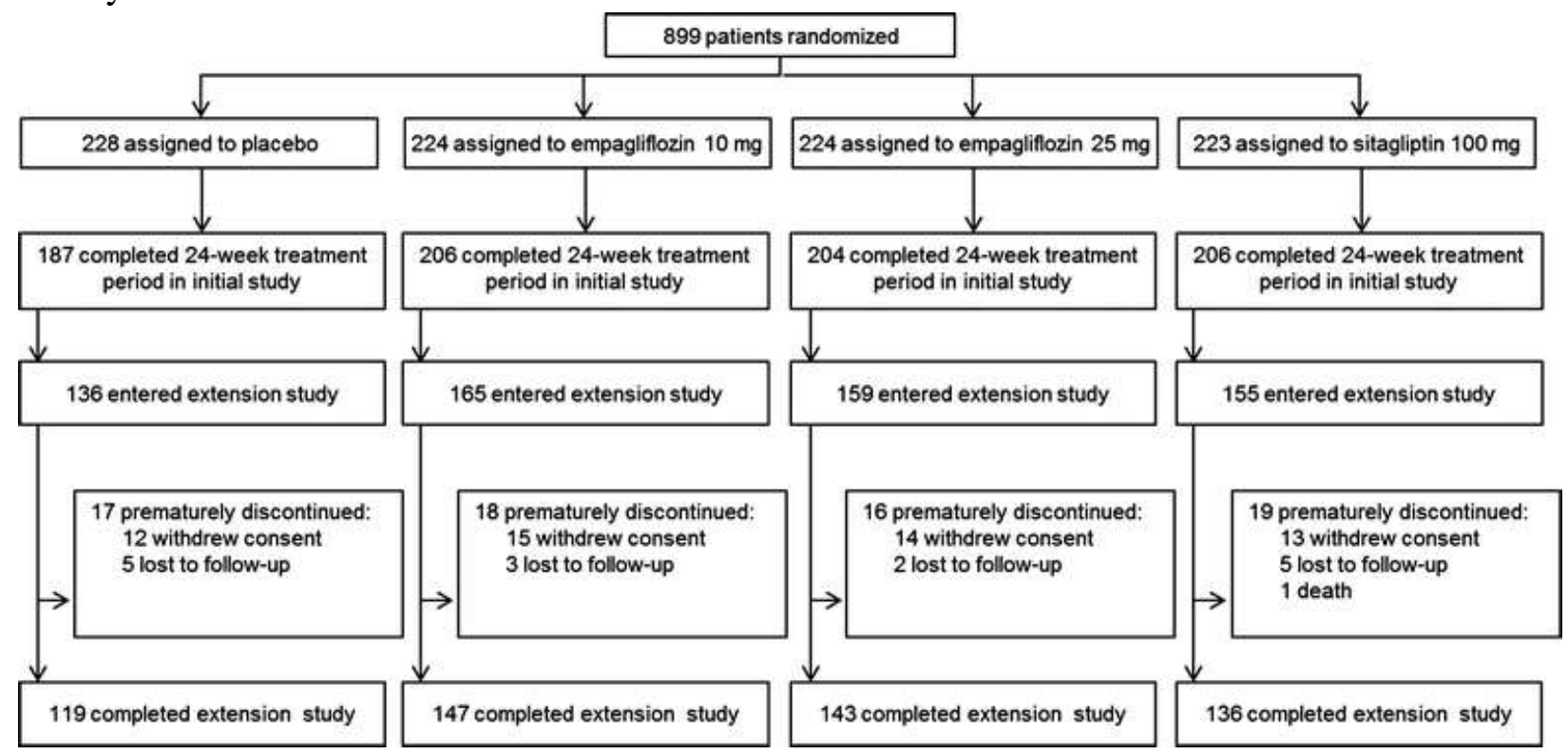

Fig 5: Application of safety audit method and risk analysis method at different levels

\section{CONCLUSION}

Subway construction process is a complex dynamic process. The occurrence of accidents is an emerging phenomenon caused by the interaction between various subjects in the system. It is not simply caused by one or more factors or events. It is necessary to analyze the dynamic characteristics of safety risks in the construction process as a whole and reveal the objective law of accidents based on the system theory and cybernetics. In the specific modeling and analysis, it is necessary to make reasonable assumptions on the analysis objective and boundary of the model according to the characteristics of the subway engineering and the actual engineering situation, which should not only reflect the characteristics of the real system, but also avoid the model being too complex due to the large boundary.

The subway construction process is a typical complex social and technical system. Accidents have both technical and organizational reasons. The safety performance of subway construction depends on the interaction between them. The safety culture of the organization determines the technical aspects of management and decision-making in safety management practice and further affects the specific 
construction operations, thus determining the size of safety risks. On the other hand, system safety performance such as safety risks will be fed back to organizations to change organizational safety culture and adjust corresponding safety management strategies through organizational learning, and system simulation analysis is a better way to describe and analyze this complex process.

\section{ACKNOWLEDGEMENTS}

This research was supported by Key Scientific and Technological Project in Henan Province [202102310576].

\section{REFERENCES}

[1] Han Xuan, Li Ning. Applicability Analysis of Peck Formula in Ground Deformation Prediction of Tunnel Construction in China. Geotechnical Mechanics, 2007, 28 (01):121-127

[2] Li Zhucai, Li Chen, Xue Yiguo. Study on the Key Technology of Comprehensive Prediction and Early Warning of Tunnel Construction Geological Hazards in High Risk Karst Area. Journal of Rock Mechanics and Engineering, 2008, 27 (007): 1297-1307

[3] Yang Xiaohua, Yu Yonghua. Application of Cement Water Glass Double Liquid Grouting in Loess Tunnel Construction. Chinese Highway Journal, 2004, 9:107-112

[4] Huang Hongwei, Zeng Ming, Chen Liang. Development of Risk Management Software for Shield Tunnel Construction (trm1.0) Based on Risk Database. Journal of Underground Space and Engineering, 2006, 2 (1): 3641

[5] He Yu, Li Fuming, Xiao Yang. Study on Advance Prediction Technology of Tunnel Construction Crossing Syncline. Journal of Underground Space and Engineering, 2020, V.16; No.126 (s1): 492-497

[6] Tan Zhongsheng, Yang Xiaolin, Wang Mengshu. Analysis of the Influence of Blasting on the Existing Tunnel in Double Track Tunnel Construction. Journal of Rock Mechanics and Engineering, 2003, 7:66-69

[7] Yang Junsheng, Liu Baochen. Ground Movement and Deformation Caused by Construction of Extrusion Shield Tunnel. Geotechnical Mechanics, 1998 (03): 10-13

[8] Wu Bo, Gao Bo. Study on Surface Settlement of Urban Subway Tunnel Construction Under Complex Conditions. China Railway Science, 2006, 27 (006): 129-131

[9] Li Yunpeng, Wang Zhiyin, Han Changling. Simulation Study on Construction Process of Small Spacing Tunnel with Different Surrounding Rock Types. Geotechnical Mechanics, 2006 (01): 11-16

[10] Wu Bo, Liu Weining, Gao Bo. Analysis of Temporal and Spatial Effects of Construction Behavior of Urban Shallow Tunnel. Chinese Journal of Geotechnical Engineering, 2004, 026 (003): 340-343 